\title{
Describing Chirality as a Pseudotensor
}

\section{Orientation dependent handedness and chiral design}

Efi Efrati and William T. M. Irvine

arXiv:1301.4460

\section{Recommended with a commentary by Jonathan V. Selinger, Kent State University}

The term "chirality" refers to the asymmetry between right- and left-handed objects. This concept was first introduced by Lord Kelvin, who wrote:

I call any geometrical figure, or group of points, chiral, and say that it has chirality, if its image in a plane mirror, ideally realized, cannot be brought to coincide with itself.

In other words, an object is chiral if it is different from its mirror image, and cannot be superposed on its mirror image through any combination of rotations and translations. Since the time of Kelvin, chirality has become a central concept in many areas of science, from microscopic to macroscopic length scales. In particle physics, it characterizes an asymmetry in the weak nuclear interaction. In organic chemistry, it characterizes molecules that have distinct isomers that are mirror images of each other. In liquid-crystal science, it characterizes phases with helical modulations, which are usually (but not always) driven by the chiral structure of the constituent molecules. In biology and medicine, it characterizes the left-right asymmetry of the human body and the different effects of chiral drugs.

In the classic definition of Kelvin, chirality is a yes-or-no question, not a quantitative question - an object either is or is not equivalent to its mirror image. Indeed, when I first began working in this field, I was told that the statement that a molecule is "slightly chiral" is absurd, like the statement that a woman is "slightly pregnant." Nevertheless, for physicists who are accustomed to working with order parameters and phase transitions, it is natural to seek a chiral order parameter, which would describe how the symmetry between an object and its mirror image is broken.

We would normally assume that such an order parameter should be a pseudoscalar, a mathematical object that has no direction and that changes sign under reflection. The magnitude of this pseudoscalar would describe how much an object is different from its mirror image, and the sign would represent which way the symmetry is broken, i.e. whether the object is right- or left-handed. As an example, for a mixture of mirror-image isomeric molecules, the chiral order parameter could be the difference of concentrations of right- and left-handed molecules. More complex examples of chiral order parameters for geometric objects are discussed in a review article. ${ }^{1}$ I have worked on constructing chiral order parameters for Langmuir monolayers, ${ }^{2}$ helical polymers, ${ }^{3}$ and bent-core liquid crystals. ${ }^{4}$

Although pseudoscalar chiral order parameters have been defined for many types of objects, these order parameters consistently have certain problems:

1. In general, it is possible to continuously transform an object into its mirror image without ever passing through an achiral state (by analogy with turning a rubber glove inside out). As that transformation progresses, the chiral order 
parameter must continuously change from a positive to a negative value, and hence must pass through zero. Hence, any chiral order parameter has false zeroes - states where the order parameter is zero even though the object is chiral.

2. For a small but important subset of chiral objects, we have some intuitive sense of handedness. As an example, the normal B conformation of DNA is a right-handed double helix. However, there is no general way to connect a positive or negative chiral order parameter with an intuitively right- or lefthanded structure.

3. Many objects are not chiral by themselves, but they become chiral by their relationship with their environment. For example, a three-dimensional statue of the letter $\mathbf{F}$ floating in space is not chiral, but it becomes chiral if it is lying on the floor. We can certainly define a chiral order parameter for the relationship of the $\mathbf{F}$ with the floor, but what can we say about the $\mathbf{F}$ by itself? Does it have some chiral susceptibility to the floor?

These types of issues inspire the current paper by Efrati and Irvine. ${ }^{5}$ They begin with the simple example shown in the figure below (from Fig. 1 in their paper), composed of two rubber sheets stretched in orthogonal directions and then glued together. This object is certainly not chiral; it has mirror planes along the two stretching directions. When the square II is cut out, it forms a saddle shape that is not chiral; it still has the same two mirror planes. However, when rectangular strips III and IV are cut out, they are chiral, forming left- and right-handed helices respectively. We could say that the relationship between the stretching directions and the cutting directions leads to chirality (just as in point \#3 above). Equivalently, we could say that each helix III or IV is composed of copies of the saddle II connected together, and the helical arrangement of these saddles leads to chirality.

Efrati and Irvine go further than that. They argue that the key geometric feature of the saddle II is how it couples flow in different directions to rotations. The direction of flow is given by a vector, and the rotation is given by a pseudovector. Hence, the relationship between the direction and rotation must be given by a second-rank pseudotensor, which they call the chirality tensor $\chi$. For the square II, this chirality tensor can be written as

$\chi=\left(\begin{array}{ccc}c & 0 & 0 \\ 0 & -c & 0 \\ 0 & 0 & 0\end{array}\right)$,

which indicates that flow along the $x$ direction induces right-handed

$(+)$ rotation about the $x$ axis, while flow along the $y$ direction induces left-handed (-) rotation about the $y$ axis. For a rectangular strip cut along any arbitrary
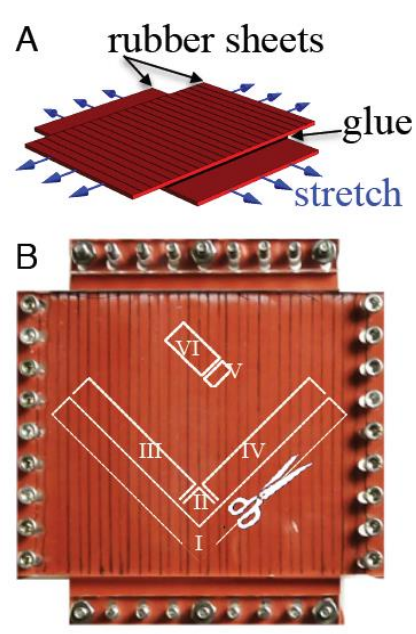

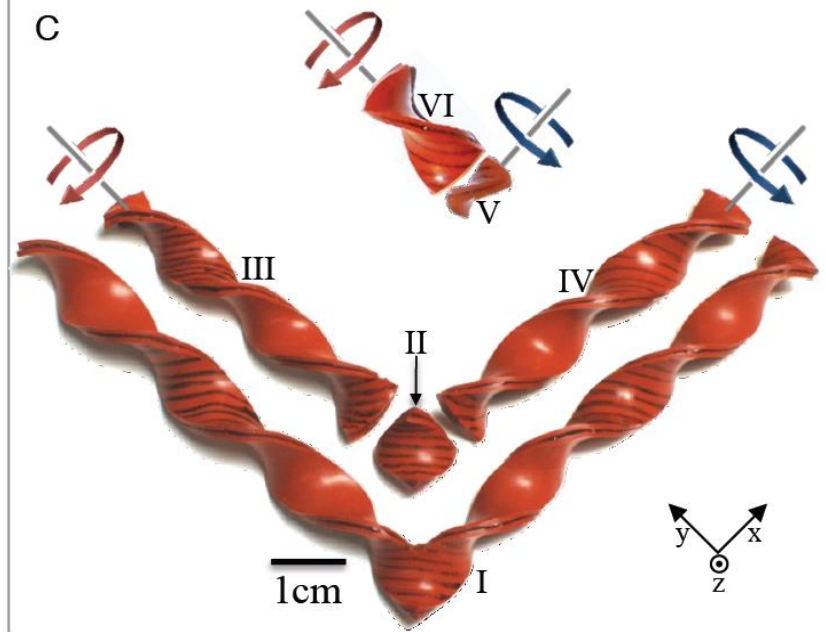


axis $\hat{n}$, the pseudoscalar order parameter can be defined as the chirality tensor contracted with $\hat{n} \otimes \hat{n}$ (which we might call the cutting tensor). As an example, strip IV has a cutting axis $\hat{n}=\hat{x}$ and hence a pseudoscalar of $+c$, while strip III has $\hat{n}=\hat{y}$ and hence a pseudoscalar of $-c$.

Efrati and Irvine provide several examples of how to construct pseudotensors to describe different types of objects, including surfaces embedded in space, 3D director fields, curves embedded in space, and metamaterials composed of semicircular wire segments. The example of metamaterials is particularly important because it involves many wires with various orientations. Each wire has a pseudotensor, which must be rotated into the correct orientation for that wire, and then the rotated pseudotensors are averaged to give the pseudotensor for the composite. This procedure illustrates how the pseudotensor approach can describe materials composed of units with a partially ordered distribution of orientations. Efrati and Irvine further argue that the pseudotensor can change sign without ever passing through zero, representing a rubber glove that turns inside out without ever passing through an achiral state.

I can imagine applying this approach to many problems in materials science where complex shapes interact to give novel behavior. For example, bent-core liquid crystals have banana-like shapes, similar to the wire segments discussed here, which couple together directions and rotations. If bent-core molecules were doped into a cholesteric helix, how would they align with respect to the helical axis and affect the pitch? One might be able to answer that question by constructing pseudotensors for the bent-core molecules and the cholesteric helix and seeing how they interact.

My main reservation about this paper concerns the terminology of "chirality tensor." It seems to me that the concept of chirality, as defined by Kelvin, is inherently isotropic rather than directional: it requires searching over all orientations to see if an object can be superposed on its mirror image. That search leads to the isotropic average over all orientations, which corresponds to taking the trace of the pseudotensor $\chi$. The full pseudotensor is a more general concept than just its trace. For that reason, I think it should have a different name-perhaps the "twist tensor" to signify that it is associated with twisted shapes that have an intuitive sense of handedness in some direction, or the "chiral susceptibility" or "pre-chirality tensor" to signify that it can be combined with another direction in space to give chirality.

A second reservation is how general this concept will be. In organic chemistry, most chiral molecules are not simple bent or helical shapes, but rather are complex irregular arrays of atoms. I'm sure it's possible to define a pseudotensor for any such molecule, but I'm not sure whether such a pseudotensor would be useful. Considering that such molecules occur in an isotropic liquid anyway, it might be better to stick with a pseudoscalar description for such systems.

Despite these reservations, I'm quite impressed with the mathematical concept that Efrati and Irvine have introduced. I look forward to seeing where it can be applied in future studies.

I would like to thank R. D. Kamien for helpful discussions, and J. M. Schnur for the example of the letter F. This commentary was supported by NSF Grant DMR-1106014.

${ }^{1}$ A. B. Harris, R. D. Kamien, and T. C. Lubensky, Rev. Mod. Phys. 71, 1745 (1999).

2 J. V. Selinger, Z.-G. Wang, R. F. Bruinsma, and C. M. Knobler, Phys. Rev. Lett. 70, 1139 (1993).

${ }^{3}$ M. M. Green, J.-W. Park, T. Sato, A. Teramoto, S. Lifson, R. L. B. Selinger, and J. V. Selinger, Angew. Chem. Intl. Ed. 38, 3138 (1999).

${ }^{4}$ J. Xu, R. L. B. Selinger, J. V. Selinger, and R. Shashidhar, J. Chem. Phys. 115, 4333 (2001).

${ }^{5}$ E. Efrati and W. T. M. Irvine, arXiv:1301.4460. 